\title{
Wind inhibition by X-ray irradiation in high-mass $\mathrm{X}$-ray binaries
}

\author{
Jiří Krtička ${ }^{1}$, Jiří Kubát ${ }^{2}$ and Iva Krtičková ${ }^{1}$ \\ ${ }^{1}$ Masaryk University, Brno, Czech Republic \\ ${ }^{2}$ Astronomical Institute, Ondřejov, Czech Republic
}

\begin{abstract}
Winds of hot massive stars are driven radiatively by light absorption in the lines of heavier elements. Therefore, the radiative force depends on the wind ionization. That is the reason why the accretion powered X-ray emission of high-mass X-ray binaries influences the radiative force and may even lead to wind inhibition. We model the effect of X-ray irradiation on the stellar wind in high-mass X-ray binaries. The influence of X-rays is given by the Xray luminosity, by the optical depth between a given point and the $\mathrm{X}$-ray source, and by the distance to the X-ray source. The influence of X-rays is stronger for higher X-ray luminosities and in closer proximity of the X-ray source. There is a forbidden area in the diagrams of X-ray luminosity vs. the optical depth parameter. The observations agree with theoretical predictions, because all wind-powered high-mass X-ray binary primaries lie outside the forbidden area. The positions of real binaries in the diagram indicate that their X-ray luminosities are self-regulated.
\end{abstract}

Keywords. Stars: early-type, stars: mass loss, stars: winds, outflows
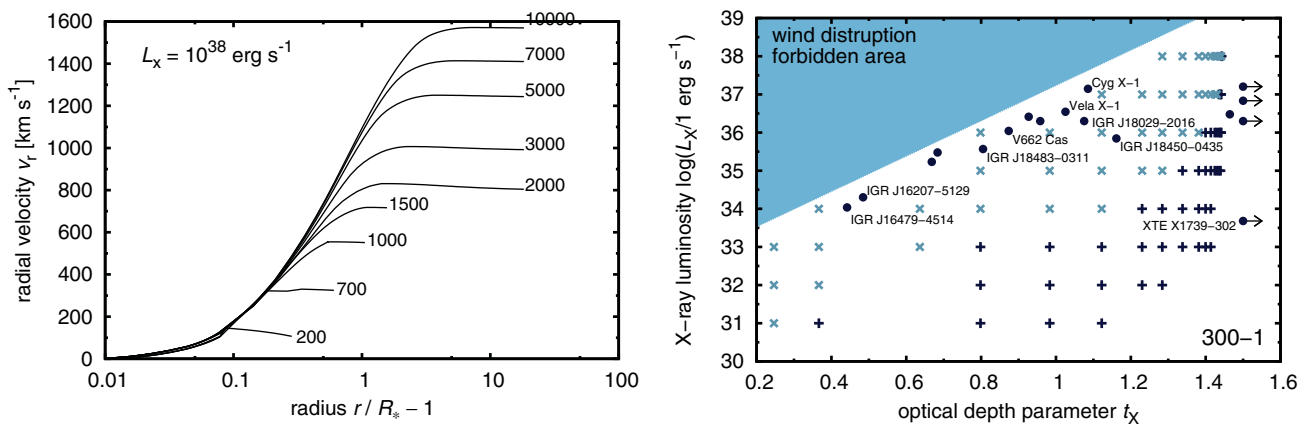

Figure 1. Left: Radial dependence of the velocity for different X-ray source distances (in $R_{\odot}$ ). The influence of X-rays is stronger for larger X-ray luminosity and lower optical depth parameter (for closer X-ray source). Too strong or too close X-ray source inhibits the flow. Right: Diagram of X-ray luminosity vs. the optical depth parameter displaying regions with different effects of the X-ray irradiation. Individual symbols denote positions of: models with negligible influence of $\mathrm{X}$-ray irradiation (black plus, + ), models where $\mathrm{X}$-rays lead to the decrease of the wind terminal velocity (blue cross, $\times$ ), and non-degenerate components of HMXBs (filled circles, $\bullet$ ).

\section{Acknowledgements}

This research was supported GA ČR 13-10589S.

\section{Reference}

Krtička, J., Kubát, J., \& Krtičková, I., 2016, AछA, 579, A111 